\title{
Students' Struggles to Explain the Atomic Behavior of Metals in a Tensile Test Lab Supported by a Molecular Dynamics Simulation
}

\section{Prof. Heidi A. Diefes-Dux, Purdue University, West Lafayette}

Heidi A. Diefes-Dux is a Professor in the School of Engineering Education at Purdue University. She received her B.S. and M.S. in Food Science from Cornell University and her Ph.D. in Food Process Engineering from the Department of Agricultural and Biological Engineering at Purdue University. She is a member of Purdue's Teaching Academy. Since 1999, she has been a faculty member within the FirstYear Engineering Program, teaching and guiding the design of one of the required first-year engineering courses that engages students in open-ended problem solving and design. Her research focuses on the development, implementation, and assessment of modeling and design activities with authentic engineering contexts. She is currently a member of the educational team for the Network for Computational Nanotechnology (NCN).

\section{Dr. Aisling Coughlan, School of materials engineering, Purdue University \\ Prof. David R Johnson \\ Dr. Kerrie Anna Douglas, Purdue University, West Lafayette}

Dr. Douglas is a Visiting Assistant Professor in the Purdue School of Engineering Education. Her research is focused on methods of assessment and evaluation unique to engineering learning contexts.

\section{Dr. Tanya Faltens, Purdue University, West Lafayette}

Tanya Faltens is the Educational Content Creation Manager for the Network for Computational Nanotechnology (NCN) which created the open access nanoHUB.org cyber-platform. Her technical background is in Materials Science and Engineering (Ph.D. UCLA 2002), and she has several years' experience in hands-on informal science education, including working at the Lawrence Hall of Science at UC Berkeley. While at Cal Poly Pomona, she taught the first year engineering course, mentored student capstone research projects, and introduced nanoHUB simulation tools into the undergraduate curriculum in materials science and engineering and electrical engineering courses. Much of her work has focused on introducing STEM concepts to broad audiences and encouraging students, including women and others in traditionally under-represented groups, to consider graduate school. Four of her former research students are currently in Ph.D. programs and a few more are in the pipeline. 


\title{
Students' Struggles to Explain the Atomic Behavior of Metals in a Tensile Test Lab Supported by a Molecular Dynamics Simulation
}

\begin{abstract}
This pilot study was designed to explore students' misconceptions concerning the atomic mechanisms of plastic deformation within the context of a laboratory exercise. Sophomore materials engineering students performed, analyzed, and compared results from both a traditional tensile test of metals and a molecular dynamics simulation tensile test of a nanowire. The latter was performed using the Nano-Materials Simulation Toolkit on nanoHUB.org. Students' responses to in-lab worksheet questions, their written lab reports, and their responses to a related exam question were qualitatively analyzed. Recommendations are made for better integration of the simulation and traditional tensile test components of the lab.
\end{abstract}

\section{Introduction}

The atomic mechanism of plastic deformation of metals is a difficult concept ${ }^{1,2}$. Students struggle to understand how, at the atomic level, dislocation formation and motion control plastic deformation.

In traditional mechanical tensile test labs, engineering students interact with macroscale samples in a physical lab, and they learn about dislocation motion and slip from their readings or from lectures. Students have the opportunity to test macroscale material properties through group collaboration and hands on experimentation, which are designed as modes of active learning. Yet students have little opportunity to directly explore the atomic-level behavior of metals. The most common classroom exposure to atomic-level behavior is through passive forms of learning. For example, instructors typically use static figures, or at best a simplified animation depicting dislocation movement. This low level of interaction with the concepts results in students having difficulty in explaining the atomic-level processes that lead to plastic deformation, and why the active slip planes are at an angle to the tensile axis. Concept sketching ${ }^{3}$, in which groups of students draw and negotiate their understanding of edge dislocation motion, is an example of a higher level engagement activity. This particular activity was shown to result in more correct answers on the Materials Concept Inventory question regarding the reason a rod forced through a smaller diameter hole has increased strength ${ }^{3}$.

The mathematical modeling and simulation tools provided on nanoHUB.org ${ }^{4}$ offer other opportunities for students to actively construct knowledge about material processes at the atomic level. The nanoHUB.org simulation tools can be incorporated into existing courses, enabling students to see and experiment at the atomic level. The move to integrate the simulation tools that researchers use to conduct nanotechnology research into the classroom is supported by recommendations from the National Research Council (2000) report, How People Learn, which endorses the integration of technology with curricular materials to support student learning 5 .

Contrary to the belief that when students are able to test materials at the atomic level through simulations they will develop a deeper understanding of dislocation motion and slip, previous 
research has not been able demonstrate the effectiveness of simulation labs in material science courses $^{6}$. Furthermore, despite the integration of a nanoscale tensile test simulation in a required sophomore level materials laboratory course for a number of semesters, our experience has been that students continue to struggle to explain plastic deformation at the atomic level, leading to the conclusion that the simulation integration with the traditional tensile test lab has not yet realized an optimal impact on students' learning. Students' continued struggles to understand atomiclevel processes highlight that the mere presence of technology in a classroom does not guarantee students' learning will be enhanced ${ }^{5}$. To improve the integration of a nanoscale tensile test simulation in a traditional tensile test laboratory exercise, how students struggle with concepts associated with the atomic mechanisms of plastic deformation in the existing lab exercise must be understood.

\section{Student Conceptual Understanding}

Students do not come into the classroom as blank slates, ready to be written on by the words of textbooks or instructors. Rather, students come into the classroom with preconceptions; new knowledge is then constructed through the filter of these prior understandings, cultural experiences, and personal beliefs ${ }^{5}$. Letting go of misconceptions is very difficult and requires that instructors design curricular activities to directly confront students' misconceptions. Given that materials behave differently at the atomic and macro-scale levels, students are particularly challenged to understand nanomaterials.

Previous research has found that, historically, material science engineering (MSE) sophomore students have difficulties understanding various materials concepts including simple theories such as stress and strain along with more difficult concepts including how a material can stretch elastically and return to its original size before yielding and how it permanently deforms plastically after the yield point. It has also been established that these students have difficulty recalling basic facts and concepts used to describe the features of a particulate system. This included students' failure to notice the structural changes governing plastic deformation in nanoscale specimens and their inability to use this knowledge to predict behavior of other materials. There are also common misconceptions concerning the relationship between elastic and plastic strain before and after yielding, as the elastic strain continues to increase after yielding for a material that work hardens.

Shown in Figure 1 are examples of tensile stress-strain curves for ductile metals corresponding to macro-scale and nano-scale specimens. The two curves shown in Figure 1a contain all the features taught in a traditional tensile testing laboratory including the definitions of Young's Modulus, yield stress, ultimate tensile stress (UTS), and percent elongation ${ }^{8}$. These curves are dominated by plastic deformation and show extensive working hardening before necking at the ultimate tensile stress. Furthermore, the differences between the annealed and cold worked (or half-hard) brass demonstrate the effect of cold work on increasing the yield stress and decreasing the ductility. Students are able to visualize the uniform plastic deformation that occurs in the tensile bar by performing hands-on tests. Figure 1(b) shows the plastic deformation from the initial metal bar length through necking (thinning of the sample's mid-section) to fracture for sample tensile bars. 
The deformation of a platinum nanowire is shown in Figures $1 \mathrm{c}$ and $1 \mathrm{~d}$. The yield stress for the nanowire ( $\sim 6000 \mathrm{MPa})$ is an order of magnitude greater than that of the macroscale tensile bars shown in Figure 1a. Additionally, the nanowire stress-strain curve shows work softening behavior as the stress decreases with further deformation after yield while the macroscale tensile bar stress-strain curve shows work hardening. The high yield stress for the nanowire is a result of the need to nucleate dislocations to initiate plastic deformation whereas multiplication of preexisting dislocations occurs in the macroscale specimen. The original nanowire is a perfect single crystal and after plastic deformation the slip planes (highlighted in white) are easily identified as shown in Figure 1d. The work softening behavior is a result of the inability of the nanowire to store dislocations and the increased ease of dislocation nucleation on the surface due the presence of a slip step.

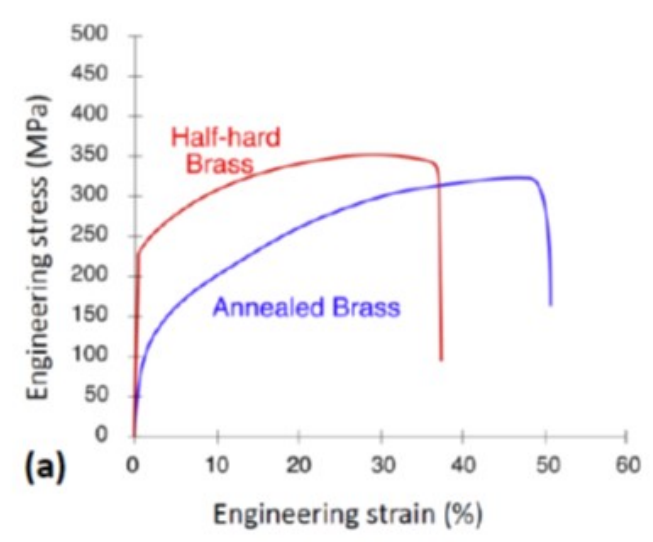

(b)
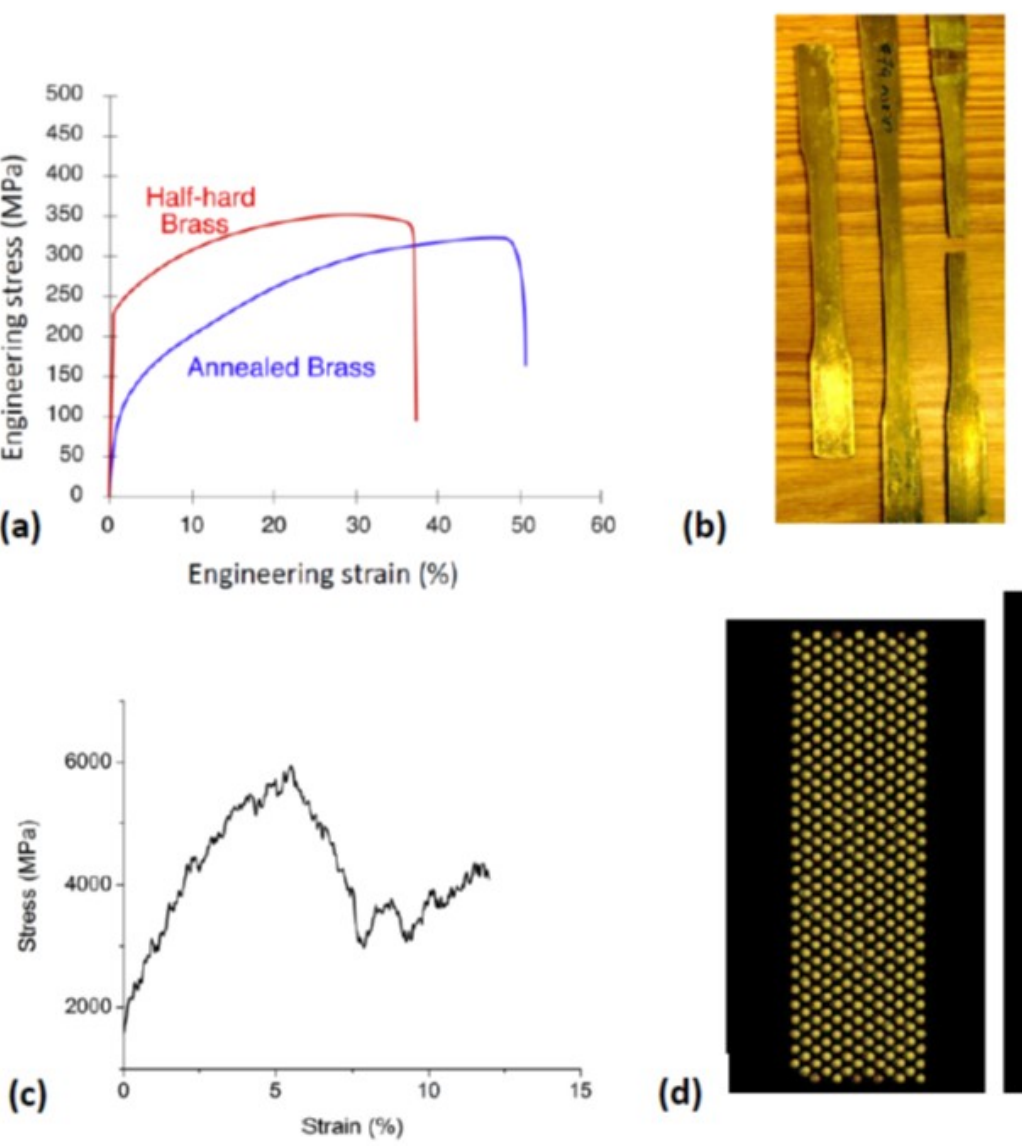

(d)
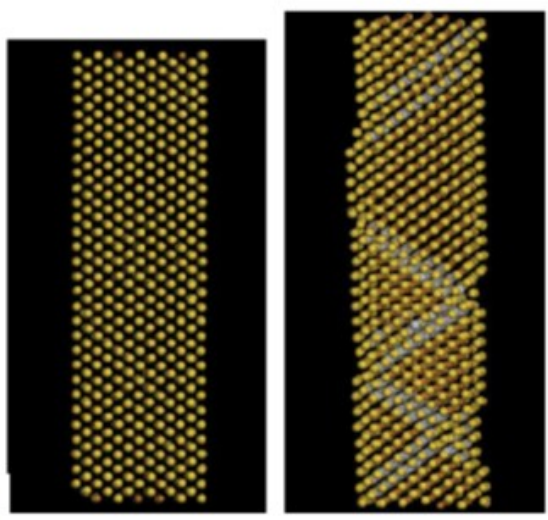

Figure 1. Stress strain curves for macro and nanoscale materials. (a) Engineering stress-strain curves for brass tensile bars, (b) Annealed and half-hard brass tensile bars before and after straining $^{8}$, (c) Stress-strain curve for a $1.3 \mathrm{~nm}$ diameter platinum nanowire from a molecular dynamics (MD) simulation, and (d) Atomic images of the wire at 0 and $12 \%$ strain. 


\section{Methods}

\section{Participants and Setting}

The setting for this pilot study is a required sophomore-level materials science engineering course. MSE 235 - Materials Laboratory - introduces students to the relationship between structure and properties of materials through both lecture and laboratory sessions. Lectures are provided before and after each laboratory session to introduce concepts and debrief, respectively. This course also provides basic tools that will be employed and built upon in subsequent materials engineering courses. Students in this course usually have either already taken or are concurrently enrolled in MSE 230 - Structure and Properties of Materials. This is due to MSE 230 being an introductory material science course detailing the structure, properties, and processing of engineering materials including the relationships between the different levels of internal structure and the basic properties, as well as the ways material structures (and thus properties) are controlled and manipulated in basic processing operations.

Thirteen students were enrolled in MSE 235 in summer 2014. Table 1 provides demographic information about these students. Two of the thirteen students enrolled in MSE 235 were repeating the course.

Table 1. Demographic information for MSE 235 enrollment in summer 2014.

\begin{tabular}{|l|c|c|c|}
\hline & Male & Female & Total \\
\hline Gender & 9 & 4 & 13 \\
\hline Repeating student concurrent in MSE 230 & 1 & 0 & 1 \\
\hline Repeating students with prior completion of MSE 230 & 0 & 1 & 1 \\
\hline Concurrently in MSE 230 & 6 & 1 & 7 \\
\hline Prior completion of MSE 230 & 3 & 2 & 5 \\
\hline No concurrent or prior MSE 230 & 0 & 1 & 1 \\
\hline
\end{tabular}

\section{Tensile Test Lab}

Since the instructors felt that molecular dynamics (MD) simulations provided a useful hands-on activity to engage students in reviewing and discussing tensile behavior and strengthening mechanisms ${ }^{6}$, simulations were directly incorporated into the tensile testing laboratory. The first integration of the simulations with the tensile lab occurred during the fall 2013 and summer 2014 semesters. The goal of adding the MD simulations into the tensile test lab was to aid students in their understanding of the atomic level processes that are responsible for the deformation responses they measure for the (macroscopic) tensile bars. As stated in the lab handout text, "By comparing the nanowire's yield stress to the yield stress of the macroscopic metal sample, you will observe the effect of sample lengthscale on mechanical behavior and relate your observation to dislocation density and motion." The specific learning objectives, also stated in the lab handout, are to enable students to:

"(i) Determine the yield strength, ultimate tensile strength (UTS), \%elongation to failure from the data they collect;

(ii) Develop an atomic picture of plastic deformation in metal; 
(iii) Understand the orientation of slip with respect to the tensile axis;

(iv) Estimate the strength of nanoscale single crystals and macroscale polycrystalline samples

(v) Understand strain hardening and the differences between annealed, cold worked, and nanoscale samples."

In this implementation, students were divided into two groups, which took turns performing standard tensile tests on macro-sized samples and running MD simulations on platinum nanowires. Results from the standard tensile tests and simulated nanowire tests were then discussed in a post-lab lecture lead by the course instructor.

Before coming to lab students were required to complete a worksheet consisting of four questions related to the tensile stress-strain curve for cartridge brass. In addition to reproducing the equations for engineering stress and strain and percent elongation (including parameters and units), students were to make a sketch of the stress-strain curve with the $0.2 \%$ offset yield stress defined using the material properties listed in their textbook. From this curve they were to show how Young's modulus was calculated. During the laboratory, students, with help from the teaching assistant, performed tensile tests on copper and brass samples. The tests resulted in force-displacement curves that the students converted to stress-strain curves using measurements of the samples' dimensions. An extensometer was used to measure displacements for small strains so that accurate values of Young's modulus could be calculated.

Students used the nano-Materials simulation to perform virtual tensile tests on platinum nanowires and analyze the results using interactive methods. The simulations are performed online using the nano-Materials Simulation Toolkit on nanoHUB.org ${ }^{7}$. This tool provides a simple and intuitive graphical interface to a research-grade molecular dynamics code denoted $\mathrm{CMDF}^{7,8}$. During the laboratory, different diameter platinum nanowires could be chosen and the temperature and strain rate could be defined by the students. Results from these tests consisted of stress vs. time curves and images of the nanowire at different times during the simulated tensile test. For the laboratory, a specific strain rate and temperature $(300 \mathrm{~K})$ were defined, but the students were encouraged by the teaching assistant to try a series of different values. An in-lab worksheet was to be completed by students during the lab and checked for completion (in-lab) by the teaching assistant. The questions were to aid the informal in-lab discussions between the students and the teaching assistant.

\section{Data Collection \& Analysis}

Three pieces of student work were analyzed for this pilot study: (1) student responses to the inlab worksheet questions, (2) relevant components of their laboratory reports, and (3) their responses to exam questions related to the tensile test lab content. During the simulation portion of the lab, students were to answer a series of questions with the guidance of the teaching assistant. These questions are listed in Table 2. Students' responses were submitted with their laboratory reports.

In the laboratory report, students were required to include three pieces related to the MD simulation. First, they were to create a figure that displays representative simulation results from a nanoscale metal nanowire being deformed in tension. Quantitative stress-strain results as well as images of the nanowire from before/after deformation were expected. Following the figure, 
the students were to compose a short paragraph describing "the what" and "the so what" of the figure, making sure to discuss all important features of the stress-strain curve. It was suggested that the results be related to dislocation density and motion. Finally, students were required to write one final short summary paragraph comparing the nanoscale and macroscale results and observations to each other, directly highlighting the differences between each testing method and describing what these differences mean.

Table 2. In-lab worksheet questions.

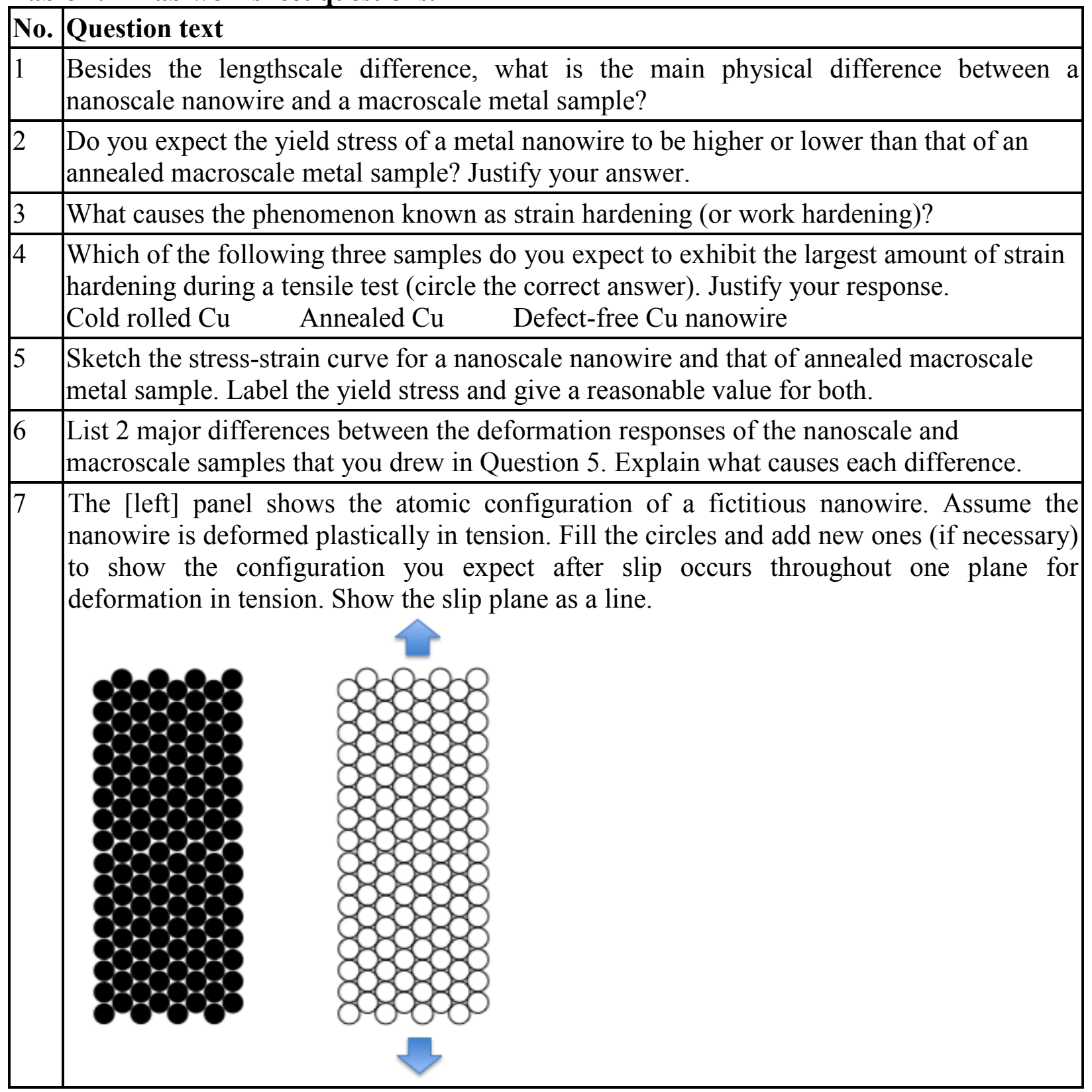

There was a series of questions on the final written exam that pertained to the tensile test lab exercise content. These questions are shown in Table 3. 
Table 3. Exam questions.

\begin{tabular}{|c|c|}
\hline No. & Question text \\
\hline 1 & $\begin{array}{l}\text { Hot rolled commercially pure copper has a Young's Modulus of } \mathrm{E}=115 \mathrm{GPa} \text {, Yield Stress } \\
\text { of } \sigma_{\mathrm{y}}=69 \mathrm{MPa} \text {, and an Ultimate Tensile Strength of UTS }=220 \mathrm{MPa} \text {. Now consider a } \\
\text { nanowire of the same material, and give a reasonable order of magnitude estimate with } \\
\text { units for each property. } \\
\mathrm{E}=\left[\quad \sigma_{\mathrm{y}}=\right.\end{array}$ \\
\hline 2 & Explain your estimate of Young's Modulus \\
\hline 3 & $\begin{array}{l}\text { What is the main physical difference between a nanoscale nanowire and a macroscale } \\
\text { metal sample that accounts of the difference in } \sigma_{\mathrm{y}} \text { ? }\end{array}$ \\
\hline
\end{tabular}

Each of these pieces of student data was qualitatively analyzed for themes in student responses. Frequency of responses with some sample quotes are provided in the results.

\section{Results}

\section{Tensile Test Lab - In-Lab Worksheet Questions}

Eleven of the thirteen enrolled students submitted a lab report. The series of in-class questions asked the students to compare the nanowire and macroscale metal samples responses to a tensile test and explain differences. While most students could correctly draw the stress-strain curves for the samples and estimate the yield stress, students had difficulty providing an atomic level process explanation. Students often pointed to the fact that physical difference between the samples is their single crystal versus polycrystal structure. Explanations of the presence, formation, and motion of dislocations were often missing, incomplete, or disjointed. The results from each of the six in-class questions are detailed in this section.

In response to question 1, regarding the main physical difference between a nanowire and a macroscale metal sample, all eleven students made reference to the crystal structure being the main physical difference between the nanowire and the macroscale metal sample. Six students specifically wrote that the nanowire is a single crystal, while five students stated that the macroscale metal sample is a polycrystal (or crystal system). Two students wrote that the "presence of vacancies" is a difference between the samples, though which sample has vacancies was not clarified. One student indicated that a macroscale metal sample "will have deformities". Two students noted the composition of the laboratory samples - the two materials were different - copper and brass (macroscale metal) and platinum (nanowire). One student noted that the size of the samples were different.

In response to question 2, concerning the relative yield stress of a metal nanowire versus an annealed macroscale metal sample, all eleven students responded that they expect the nanowire to have a higher yield stress. Two students did not provide a justification for this expectation. Three students referred to only the difference in the crystal microstructures (single vs. polycrystalline) of the samples. Four students mentioned the presence or absence of dislocations typically while mentioning the crystal structure. These students provided no or little further 
explanation. Two students identified that for the nanowire dislocations need to be created and/or that dislocations in the macroscale metal sample need only be moved.

When describing what causes the phenomenon known as a strain hardening (question 3), one student seemed to attempt to define strain hardening as "The increasing of strain in a multicrystal structure." Five students responded that the phenomenon is related to plastic deformation, with little or no further explanation. Of these, two students noted that the strain hardening that occurred during testing would subsequently lead to a higher yield stress. For instance, one of these two students wrote, "Yield strength increases after plastic deformation, causing the material to be stronger." Three students related strain hardening to an increase in the number of dislocations which create obstacles to deformation. Two students identified that the dislocation density increases, though they did not explain how this causes strain hardening.

Ten of the eleven students selected annealed copper as exhibiting the largest amount of strain hardening during a tensile test (question 4). Four students described how annealing results in filling in the dislocations or removing the vacancies. Two students compared the number of dislocations present in cold-rolled and annealed copper, though they split the decision on which treatment actually resulted in more dislocations. One wrote that annealed copper "is a polycrystalline material but with the least amount of dislocation, this gives it the greatest potential to be hardened", while the other stated, "annealing is better than cold rolled because there are more deformities per section of material." Two students eliminated the nanowire from contention by stating that the nanowire undergoes little or no strain hardening.

For question 5, all eleven students sketched a correct stress-strain curve for a nanowire, including the noise that is an artifact of the computations performed by the simulation. All eleven students sketched a correct stress-strain curve for a macroscale metal sample, though one student did not draw the region corresponding to necking of the macroscale sample. Two students provided no yield stress values. Nine students had yield stress values marked in the correct location on the stress-strain curve (at the end of the linear region) and in the range of 1000 to $6000 \mathrm{MPa}$ for the nanowire. Eight students had a yield stress in the range of 50 to $500 \mathrm{MPa}$ for the macroscale metal sample; one student's value was difficult to discern (perhaps $1 \mathrm{~Pa}$ ). Seven of the eight students marked the yield stress at the end of the linear section of the curve; the eighth student marked it at the UTS.

For question 6, six of the eleven students listed differences between the deformation responses of the nanoscale and macroscale samples without attempting to explain the differences. These students noted (or described):

- the nanowire has a higher yield stress than the macroscale metal sample $(n=4)$

- strain softening of the nanowire $(n=4)$

- strain hardening of the macroscale metal sample $(n=4)$

- the stress-strain curve is different after the yield stress is reached $(n=1)$

- the nanowire's stress-strain curve oscillates while the macroscale metal sample's stress-strain curve is smooth $(n=1)$

Of the five students who tried to explain the differences, two students simply explained that the nanowire has a higher yield stress than the macroscale metal sample because it is a single crystal. The students who attempted to explain the differences discussed the presence or absence of 
dislocations. For example, "The [yield strength] for the nanoscale sample is much higher than for the macroscale sample because it doesn't have voids or flaws. It takes much more stress to deform the nanoscale sample." Others discussed the formation and movement of dislocations. For instance, one student wrote, "In nanoscale, an increase in deformation forms a dislocation which will move to the edge and eventually disappear. In macroscale, the number of dislocations increase which makes it more resistant to deformation. The disappearance of dislocations reduces the stress in a nanowire. Whereas in a macroscale, stress increases making the material stronger." Another student wrote, "In macroscale stress moves a dislocation. The number of dislocations increases. In nanoscale, stress produces a dislocation and dislocation move to the edge and disappear." And still another student wrote, "The nanowire is a perfect crystal, so dislocations can move out." Two students indicated something similar to what this student wrote, "after yield stress is reached, the nanoscale sample fails immediately while the macroscale sample goes through plastic deformation first - this is due to the fact that the nanoscale only measures until the first distortion occurs."

Finally, for question 7, two students' atomic configurations suggest that atoms are eliminated from the edge of the sample and moved to the top and bottom of the sample. Four students had drawn a 45 degree line through the configuration with no or a variety of indications of where atoms move to. Only one of these students indicated that the atoms move along the 45 degree plane. The remaining four students provided a configuration that was consistent with that shown in Figure 2d.

\section{Tensile Test Lab - Lab Reports}

Students were asked to place in their reports stress-strain curves and images capturing the atomic structure before and after the yield stress was reached. The images many students included in their reports and the lack of discussion surrounding their results suggest they were unsure of how to analyze the MD simulation output. Students also seemed unsure of what to focus on when comparing their nano- and macro-scale tensile results.

The curves generated by the MD simulation are actually stress-time curves (for a constant strain rate); students were not asked to generate stress-strain curves. Four students included simulation results for the Pt nanowire at an elevated temperature (e.g. $2100 \mathrm{~K}$ ) in addition to $300 \mathrm{~K}$ (Figure 2), though two of these students did not acknowledge that temperature was the difference between the two simulation runs. Five students included other plots that the MD simulation generates (e.g. temperature versus time, energy versus time). Some atomic images captured by the students showed the formation and movement of dislocations better than others. The quality of the atomic image capture is a function of the angle of the image on the screen (Figure 3). 


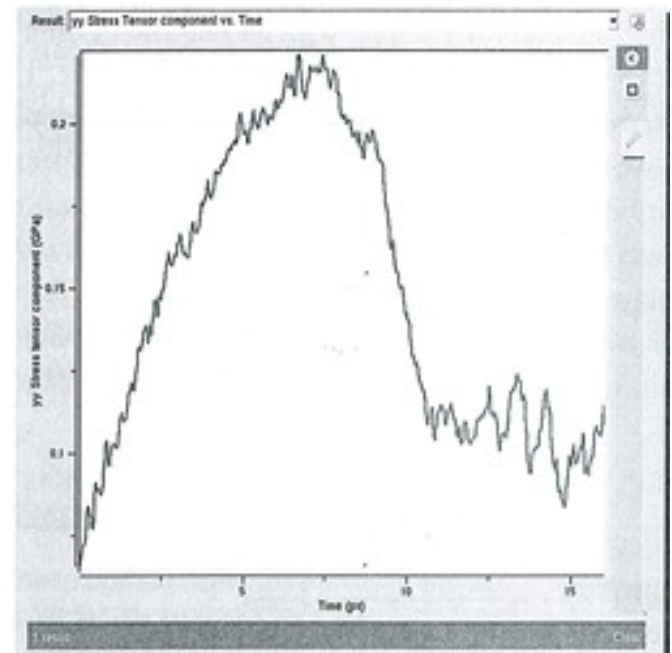

(a)

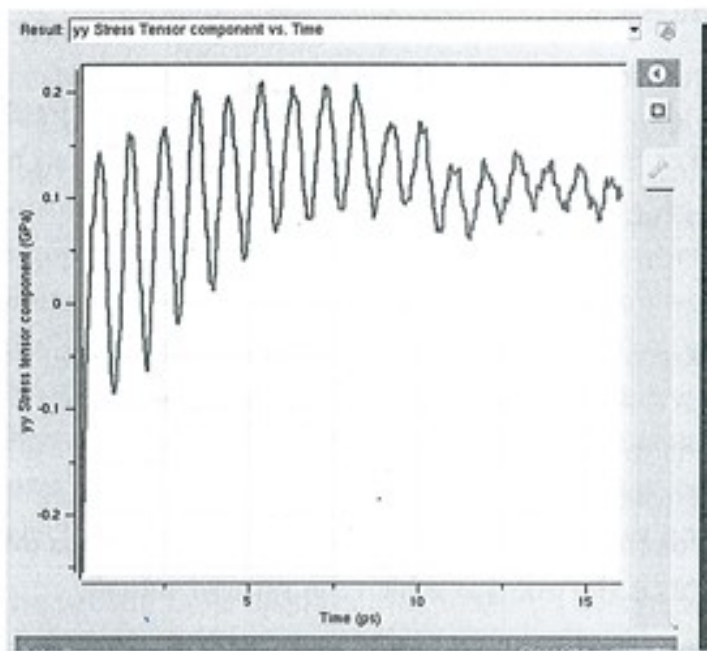

(b)

Figure 2. Stress-time curves for the Pt nanowire tensile test simulation at $300 \mathrm{~K}$ (a) and $2100 \mathrm{~K}$ (b).

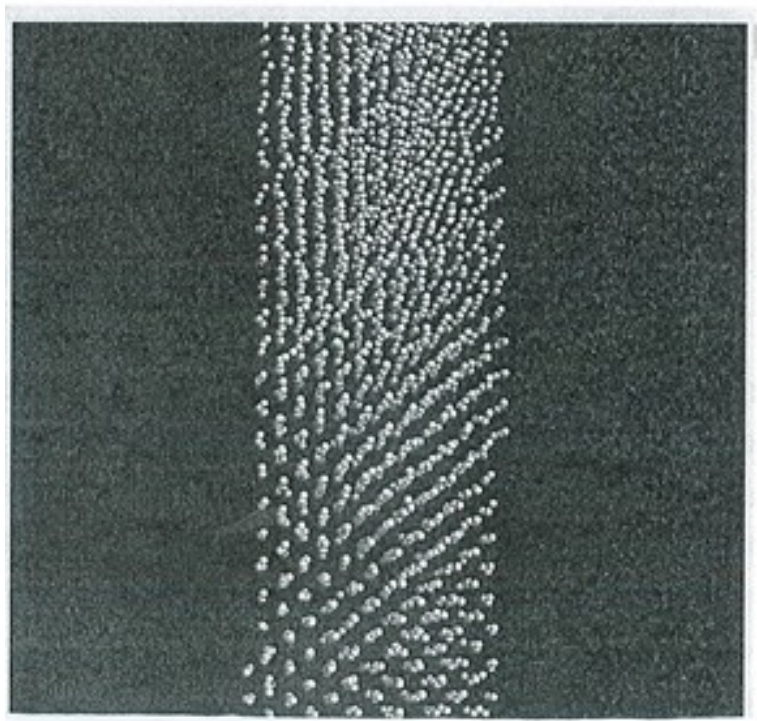

(a)

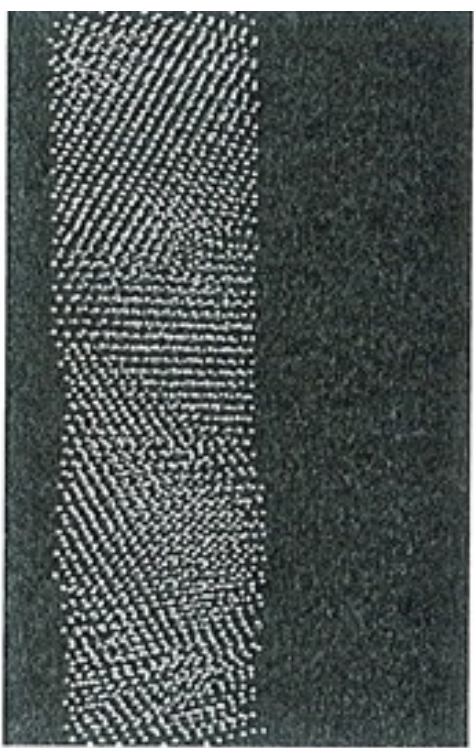

(b)

Figure 3. Atomic images for nanowire tensile test simulation after yield stress The sample is oriented differently in images (a) and (b).

If the stress-time curve results for the simulation were described, students pointed out only one of the following: the high yield stress, the oscillations in the graph, or the point at which the first dislocation occurs or strain softening begins. Only two students described the results shown in their atomic images. Both pointed to the images showing evidence of slip.

When students compared the macro- and nanoscale test results, no student did a thorough comparison of the characteristic features of the stress-strain curves. That is, they did not explicitly call out differences or similarities in the values of the Young's modulus, the yield 
strength, the ultimate tensile strength, or the segments of the stress-strain curve indicating the elastic and plastic regions. Nor did any student provide a complete atomic explanation for any similarities or differences. Students attended to a variety of things including strain softening versus hardening $(n=4)$, crystal structure $(n=5)$, yield stress $(n=3)$, dislocation motion $(n=3)$, dislocation formation $(n=2)$, number of dislocations $(n=2)$, dislocation density $(n=1)$, UTS (1), post UTS stress-strain curve behavior $(n=1)$, and noise in the nanoscale stress-time curve $(n=1)$.

\section{Exam Questions}

Eleven students took the written exam analyzed here. The two students who did not submit a lab report were not the same two students who did not take the exam. The exam questions again asked the students to compare the nanowire and macroscale metal samples responses to a tensile test and explain differences. Similar to the in-class questions, most students are able to correctly make quantitative comparisons between the samples but their explanations often stop at pointing out the physical differences in the samples. Few students are able to provide complete atomic level explanations.

Young's Modulus. When estimating the Young's Modulus for the nanowire, two students correctly identified that the nanowire and the bulk copper sample would have the same Young's Modulus. One student stated that Young's Modulus is a material property and therefore, "It will be the same of a material of same composition regardless of dimensions." The second student referred to Young's Modulus as being "the elastic change of the region which is the stretching of the bonds before movement so the bonds would stretch the same distance in the same sort of material no matter what form." These students correctly identified the region of the stress-strain curve where Young's Modulus is measured and that elongation at the atomic level is the result of bonds stretching, as opposed to breaking and reforming.

Seven students provided Young's Modulus values that were higher than the bulk copper metal value of $115 \mathrm{GPa}$. The range of student provided values was $200 \mathrm{GPa}$ to $115 \times 10^{12} \mathrm{~Pa}$. Two students made reference to the nanowire being a single crystal. One student explained that "deforming it would mean shifting atomic bonds, which would mean that it is stiffer". Based on these students' high Young's Modulus value and the focus on the crystal structure, these students seem to be confusing the explanation for the Young's Modulus with an explanation for differences in the yield stress, which should be higher for the nanowire than the bulk copper sample.

Four students with high Young' Modulus (E) values made reference to their yield stress values, which for all of these students had the correct order of magnitude (GPa). One student generally stated that the "yield stress of nanowire will be higher under the same strain, therefore E must increase as well." The other three students provided a computational- based explanation. They used the yield stress and the Young's Modulus value for the bulk copper metal to calculate strain $(\varepsilon)$ to the yield stress $\left(\sigma_{\mathrm{y}}\right)$ point using $\mathrm{E}=\sigma_{\mathrm{y}} / \varepsilon$. Then, used their equation, plugging in their calculated bulk copper $\varepsilon$ and their estimate of yield stress value for the nanowire, to compute $E$ for the nanowire. The students understand how to use the information provided to compute E. However, a few potential misconceptions are apparent. They are missing the concept that Young's Modulus is a material property and they are not expressing that they have a sense of the atomic mechanism by which elastic deformation occurs. They are, perhaps unintentionally, 
indicating that the strain needed to achieve the yield stress should be the same for both samples. Or it could be that they do not realize that materials with the different yield stresses can have the same Young's Modulus, which in turn means that it takes different strains to get to the yield stress point. This could be a graphing misconception related to understanding slope given two points on a line, in this case the stress-strain curve: $(0,0)$ and (strain,yield stress). It could be they are not making a connection between $\mathrm{E}=\sigma_{\mathrm{y}} / \varepsilon$ and the stress-strain curve at all.

One student's response was a combination of the two types of responses described above. The student pointed to the single crystal structure of the nanowire and then stated of the nanowire, "they are harder to elongate. Therefore you will have a larger stress for less strain causing E to be bigger."

Two students provided slightly lower values of the Young's Modulus than the provided bulk copper value. One student referred to the "Elastic Modulus" being the slope of the curve but then stated "since the nanoscale wire decreases it would be smaller". This student drew correct general representation of the stress-strain curve for both nanoscale and macroscale samples. It is likely the "decrease" refers to the plastic region of the nanoscale curve. The other student acknowledged that the Young's Modulus values "should not be very different" (student responded with $100 \mathrm{GPa}$ ) and the "nanowire sample can withstand more stress, but it must also undergo a large amount of strain to reach the $\sigma_{\mathrm{y}}$." So, this student understands that a larger strain is needed to achieve the yield stress for the nanowire.

Overall, few students provided an atomic-level explanations for their Young's Modulus value. This may, in part, be due to the phrasing of the question that does not direct students towards this type of explanation.

Yield Stress. Nanowire yield stress $\left(\sigma_{\mathrm{y}}\right)$ values with an order of magnitude in the GPa range are typical (Figure 1(c)), and depending on the type of materials being tested the bulk material yield stress values range from 50 to $500 \mathrm{MPa}$ (Figure 1(a)). Student responses in the GPa range fell into three groups: 1-2 GPa $(n=4), 10-40 \mathrm{GPa}(n=3)$, and 100-600 GPa $(n=2)$. One student response was in the $\mathrm{MPa}$ range, $690 \mathrm{MPa}$, and one student response had an unclear order of magnitude. Most students identified the correct order of magnitude for the nanowire.

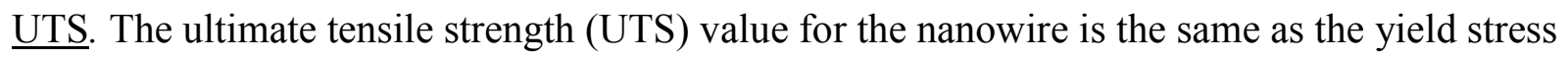
value, as dislocations move to the free surface to reduce the strain energy and the nanowire strain softens. Of the nine students that had provided yield stress values in the GPa range, five students provided the exact same UTS value. The other four students provided a variety of responses. One gave a UTS value that was slightly higher $\left(\sigma_{\mathrm{y}}=1 \mathrm{GPa}, \mathrm{UTS}=1.2 \mathrm{GPa}\right)$, perhaps not realizing that strain softening immediately follows attainment of the yield stress. One student gave a value that was much higher and exactly matched to their estimated Young's Modulus $\left(\sigma_{\mathrm{y}}=10 \mathrm{GPa}\right.$, $\mathrm{E}=\mathrm{UTS}=100 \mathrm{GPa}$ ), this might indicate student confusion over what these values are measuring. One student provided a UTS value that was significantly lower than their yield stress and Young's modulus values $\left(\sigma_{\mathrm{y}}=600 \mathrm{GPa}, \mathrm{E}=60 \mathrm{GPa}, \mathrm{UTS}=100 \mathrm{MPa}\right)$. One student did not believe that a nanowire would have a UTS value, stating that the "material fractures before a noticible strain occurs". The student with the low yield stress value of 690 MPA did have a matching UTS value. The student with the indecipherable yield strength value had a low UTS value $\left(220 \times 10^{4} \mathrm{~Pa}\right)$. 
Physical Difference Between Nanowire and Macroscale Metal. When responding to the prompt concerning the physical difference between the nanowire and the macroscale metal that would account for differences in the yield strength, students made reference to one or two main ideas: (1) the crystal microstructure (single or polycrystalline) and (2) the presence or absence of dislocations. Ten students made reference to the nanowire being a single crystal; two of these students left their explanation at this. Six students went on to refer to the macroscale metal having a polycrystalline structure; one of these students left their explanation there. One student made a general reference to the crystalline structure being at the root of the difference but did not indicate how the nanowire and macroscale metal are different in this regard, though this student did go on to discuss dislocations.

Seven students made reference to dislocations (also termed defects or imperfections by the students). Two students focused on their presence or absence without further explanation. The student that referred to imperfections seemed to regard these as different from dislocations, stating "macroscale samples already have dislocations and imperfections to act as stress concentrators"; this student also made reference to the lack of imperfections in the nanowire as making it "much more stable".

Two students indicated for the nanowire that dislocations must be created whereas for a macroscale metal, the dislocations must be moved, with one of these students clarifying that "The creation of a dislocation require higher stress than moving a dislocation."

The following are the only examples of students attempting an atomic level explanation for the difference in the yield stress:

The first student wrote, "The nanowire is a single crystal, so it does not exhibit plastic deformation from grains moving against one another like in a polycrystalline macroscale sample. On the contrary, the nanowire is just one grain. This gives the atoms more freedom to move farther elastically from one another." While this student realizes that the nanowire is a single crystal and the macroscale tensile specimens are polycrystalline; however, learning objectives related to (ii) Develop an atomic picture of plastic deformation and (iii) Understand the orientation of slip with respect to the tensile axis were clearly not meet. A discussion of dislocation motion and the observation of single slip would show knowledge of the atomic level mechanisms that are responsible for plastic deformation in both single and polycrystalline metals.

The second student wrote, "nanoscale wire is basically a single crystal whereas a macroscale metal sample is comprised of an entire crystal system. This crystal system lends itself to the phenomena of strain hardening, which moved dislocations, increases hardness, and as such $\sigma_{\mathrm{y}}$. nanoscale wire experiences phenomena of strain softening." This student has recalled some of the discussion that took place during the laboratory, but was unable to complete the analysis when writing the report. The student is using the confusing term "crystal system" to describe the polycrystalline microstructure of the macroscale tensile specimen. However, the student does indicate that plastic deformation is related to dislocation motion and that strain hardening is related to the presence of dislocations. These are fairly advanced topics to be discussed in the first tensile lab. Microstructures, the measurement of grain size, and strengthening mechanisms, 
including strain hardening and dislocation density, are introduced in separate labs later in the semester. The initial discussion in this laboratory provides some context for the subsequent labs.

The third student wrote, "A nanscale nanowire is a single crystal. Therefore it will have a high $\sigma_{\mathrm{y}}$ because the stress needed to break its bonds. A macroscale sample has dislocations, and it will fail at its dislocations, not by breaking bonds. This required less energy, so macroscale samples have lower $\sigma_{\mathrm{y}}$ values." This student also seems to recall some of the discussion that took place during lab but cannot reformulate the key ideas. The reason the nanowire does not contain dislocations is related to the specimen being so small that it is dislocation-free, and as a consequence, the specimen is also a single crystal. The student knows that plastic deformation is related to dislocations but cannot describe the atomic level processes related to the motion (the local breaking and reforming of bonds).

\section{Discussion}

It is apparent from students' responses to the in-lab worksheet questions, their lab report documentation, and their responses to the exam questions, that students struggle to articulate the atomic level processes occurring when different materials are under strain. Evidence suggests that students do have some knowledge in relation to the order of magnitude of Young's modulus and yield stress for both nanoscale and macroscale materials. They can also state that the nanomaterial, a platinum nanowire, is a single crystal and a macroscale material, bulk copper, is polycrystalline. The majority of students can also state that the former has no dislocations initially and the latter has many dislocations. The key areas where students tend to struggle are describing the relationship that exists between the existence, formation, and motion of dislocations and the stress-strain behavior.

To break this down even further, while all students that submitted lab reports knew that there was a microstructural difference (single vs. polycrystalline) between samples, only half correctly identified the nanowire as being a single crystal. Furthermore, only a third of the students attempted to describe dislocations. In their retrospective lab reports, all students were able to identify that the yield stress of the nanowire will be greater than that of the macroscale metal sample. However, some students related the higher yield stress of the nanowire to the fact that it was a single crystal rather than to it being defect free. For example, only two students correctly described the need to nucleate dislocations in the nanowire as the reason for the high yield stress, whereas the yield stress in the macroscale metal samples was due to the motion of dislocation already present in the sample.

Since students had difficulty in relating dislocations to the yielding phenomenon, their difficulty in articulating the impact of cold-rolling and annealing in relation to the presence of dislocations (and the dislocation density) is not surprising. This is apparent as in their lab reports they failed to mention that the presence of dislocations actually results in strain hardening. A possible explanation as to why students have difficulties with the appearance, movement, and disappearance of dislocations in single and polycrystalline materials is that during the laboratory sessions, dislocations are not directly observed in the macroscale specimens and are rarely captured in the structural output from the MD simulations (Figure 3). What are shown in these figures are the slip traces produced after the dislocations shear the crystal. 
The overall impression garnered from student lab reports is that students form incomplete ideas about the role dislocations play in crystalline plasticity. One problem in the current version of the laboratory may be that too many new concepts are introduced together. Above and beyond content related to the tensile test methods and the analysis of stress-strain curves, these new concepts include microstructure (single vs. polycrystalline metals), dislocations and their movement and multiplication, and strain hardening. As the students performed the MD simulations in the laboratory, there was discussion between students and the instructor concerning the differences in behavior between the nanowire and the macroscale tensile specimens. However, students performed no quantitative analysis using the simulation results for their reports. A reorganization of the laboratory is recommended to focus on fewer topics but covered in more detail.

\section{Recommendations}

This pilot study yielded four recommendations for improving the integration of the MD simulation in the tensile test lab exercise.

1. Reduce the number of concepts included in the lab. Have the students focus on the reasons why there is a difference between the yield stress of the nanowire and the macroscopic tensile specimen and delay the discussion of work hardening (or softening) behavior until a later laboratory exercise.

2. Have students pull in relevant concepts from previous lectures and labs. The students have had previous laboratories and classes on crystallography and can apply what they have learned here. If the orientation of the platinum nanowire is given, the students can find and calculate the orientation of the slip planes and directions for the FCC crystal. The slip planes that are predicted can be compared to those observed in the MD simulations.

3. For easier comparison of the nanowire and macroscale specimen tensile test results, the students should calculate the tensile stress-strain curve for the nanowire from the simulation results and then measure Young's modulus and the yield stress. By quantitatively analyzing the stress-strain curve, students can directly compare the Young's modulus of the nanowire to that of the macroscale specimens and see that they are indeed similar. Comparison of the yield stresses will show them to be dramatically different.

4. Introduce the concepts of dislocations earlier. For example, the structure of dislocations could be introduced in the earlier crystallography lab while the basic properties could be introduced in the lecture component of the course before the tensile testing laboratory.

Overall, these recommendations require a revisit of the learning objectives and realignment of the tensile test lab activities and assessments to the learning objectives as suggested by the framework for integrating nanoHUB.org computational simulation tools into engineering lessons ${ }^{11}$. 


\section{Conclusion}

A baseline understanding of students' struggles to explain the atomic mechanism for plastic deformation was the aim of this preliminary study into the integration of a molecular dynamics simulation into a traditional tensile test lab. Students' ability to use the presence or absence of dislocations or the formation and motion of dislocations as the basis for explanations for difference in macroscale and nanoscale sample stress-strain behavior varied, but was generally limited and fragmented. Recommendations for better integration of the lab components focus on a reduction of concepts in the tensile test lab, increased analysis of simulation data for easier comparison across samples, and re-sequencing of topics before and after this the tensile test lab.

\section{Bibliography}

1. Krause, S., Decker, J., Niska, J., \& Alford, T., \& Griffin, R. (2003). Identifying student misconceptions in introductory materials engineering courses. Proceedings of the $110^{\text {th }}$ ASEE Annual Conference \& Exposition, Nashville, TN.

2. Krause, S. Tasooji, A., \& Griffin, R. (2004). Origins of misconceptions in a Materials Concept Inventory from student focus groups. Proceedings of the $111^{\text {th }}$ ASEE Annual Conference \& Exposition, Salt Lake City, UT.

3. Krause, S., \& Tasooji, A. (2008). An intervention using concept sketching for addressing dislocation-related misconceptions in introductory materials classes. Proceedings of the $115^{\text {th }}$ ASEE Annual Conference \& Exposition, Pittsburg, PA.

4. Klimeck, G., McLennan, M., Brophy, S. P., Adams III, G. B., \& Lundstrom, M. S. (2008). nanohub. org: Advancing education and research in nanotechnology. Computing in Science \& Engineering, 10(5), 17-23.

5. National Research Council. (2000). How People Learn: Brain, Mind, Experience, and School: Expanded Edition. Washington, DC: The National Academies Press.

6. Brophy, S. P., Magana, A. J., \& Strachan, A. (2013). Lectures and simulation laboratories to improve learners' conceptual understanding. Advances in Engineering Education, 4(2). Retrieved from http://advances.asee.org/?publication=lectures-and-simulation-laboratories-to-improve-learners-conceptualunderstanding

7. Coughlan, A., Johnson, D., Diefes-Dux, H.A., Douglas, K.A., Erk, K., Faltens, T.A., \& Strachan, A. (2015). Enhanced learning of mechanical behavior of materials via combined experiments and nanoHUB simulations: learning modules for sophomore MSE students. MRS Proceedings, 1762, mrsf14-1762-aaa06-03. (DOI:10.1557/opl.2015.152)

8. Bowman, K. (2004). Mechanical Behavior of Materials. New York: John Wiley \& Sons, Inc. p. 23.

9. Strachan, A., Palaria, A., Zhou, Y., \& Jhaveri, J. (2014). nano-Materials Simulation Toolkit. Retrieved from https://nanohub.org/resources/matsimtk (DOI: 10.4231/D3416T079)

10. Lin, K.-H., Holian, B. L., Germann, T. C., \& Strachan, A. (2014). Mesodynamics with implicit degrees of freedom. Journal of Chemical Physics. 141(6). (DOI: 10.1063/1.4891308)

11. Douglas, K.A., Faltens, T.A., Diefes-Dux, H.A., Coughlan, A., \& Johnson, D.R. (2015). A framework for integrating research grade simulation tools into engineering lessons. Proceedings of the $122^{\text {nd }}$ ASEE Annual Conference and Exposition, Seattle, WA. 